\title{
IDENTIFICATION OF COUNTERFEIT MEDICINES FOR ERECTILE DYSFUNCTION FROM AN ILLEGAL SUPPLY CHAIN
}

\author{
Siniša TOMIĆ, Neven MILČIĆ, Milenko SOKOLIĆ, and Adrijana ILIĆ MARTINAC \\ Agency for Medicinal Products and Medical Devices, Zagreb, Croatia
}

Received in March 2009

Accepted in August 2009

\begin{abstract}
The appearance of counterfeit medicines in supply chains is a global public health problem that may seriously affect patients. Counterfeit drugs do not meet quality standards and do not declare their real composition and/or source for the purposes of fraud. They may be generic or innovative, they may contain genuine constituents in a fake packaging, or wrong ingredients, or inactive ingredients, or an incorrect quantity of the active substance. In Croatia, no cases of counterfeit medicines have been detected so far, but the Agency for Medicinal Products and Medical Devices has received 34 samples of medicines and other products for testing from Zagreb City Police. The samples included medicines for erectile dysfunction: sildenafil, tadalafil, and vardenafil. Twenty-three samples of tablets without marketing authorisation in Croatia were tested with high-performance liquid chromatography (HPLC) for the declared sildenafil and tadalafil content. Samples labelled 1 (batch T/33), 3 (batch T/33), 5 (batch 4), 6 (batch M0016J), 10 (batch T-070235), 12 (batch T-070544), 15 (batch 314833201), 16 (batch 832718474), and 17 (batch 504830028) containing sildenafil and samples labelled 20 (batch 070356), 21 (batch 05668), and 22 (batch T 378 5) containing tadalafil did not contain the active substance within the acceptable $95 \%$ to $105 \%$ margin of deviation from the declared content. While most samples cannot be described as fake with a reasonable amount of certainty, there is still a suspicion of counterfeit. A correct conclusion can be drawn only with the assistance of the manufacturers and by conducting additional laboratory tests.
\end{abstract}

KEY WORDS: HPLC, pharmaceutical crime, quality control, sildenafil, tadalafil, vardenafil

According to the World Health Organization (WHO) definition, counterfeit medicines form part of a broader category of pharmaceutical products which do not meet quality standards in force, with the difference that they are deliberately and fraudulently mislabelled with respect to their real composition and/or source. Counterfeit medicines may be both branded and generic and may contain genuine constituents in a fake packaging, or wrong ingredients, or inactive ingredients, or an incorrect quantity of the active substance $(1,2)$. The use of such medicines poses a health risk in that the intended effect of a medicinal product is not achieved, which may result in unexpected adverse effects, anaphylaxis, resistance to medicinal product, or other health problems (3$5)$. Most industrialised countries with an efficient system and market surveillance regulation have a low percentage of such products, mostly below $1 \%$, whereas in many countries of Africa and parts of Asia and Latin America the market share of such products exceeds $30 \%(6-10)$. Counterfeit medicines in the European Union are mostly the "lifestyle" drugs, including medicinal products for erectile dysfunction and obesity (11). Apart from these, there are fake medicines for oncological, cardiac, psychiatric, and infectious diseases (12-14). This trend 
in pharmaceutical crime may further rise, because the driving force behind it are high gains and high sales accompanied by utter disregard for the health of patients, which makes it a global problem (15-18).

In Croatia, no cases of counterfeit medicines have been detected so far in licensed pharmacies or in wholesale chains. But on the illegal drug market 34 samples have been seized. The products were classified in three groups, the first of which included products authorised for marketing in Croatia, the second comprised products without marketing authorisation, and the third products which are not medicines but rather medical devices or dietary supplements (Figure 1). The first and the second group consisted of 26 samples indicated for the treatment of erectile dysfunction, declared to contain active substances sildenafil, tadalafil, or vardenafil (Figure 2).

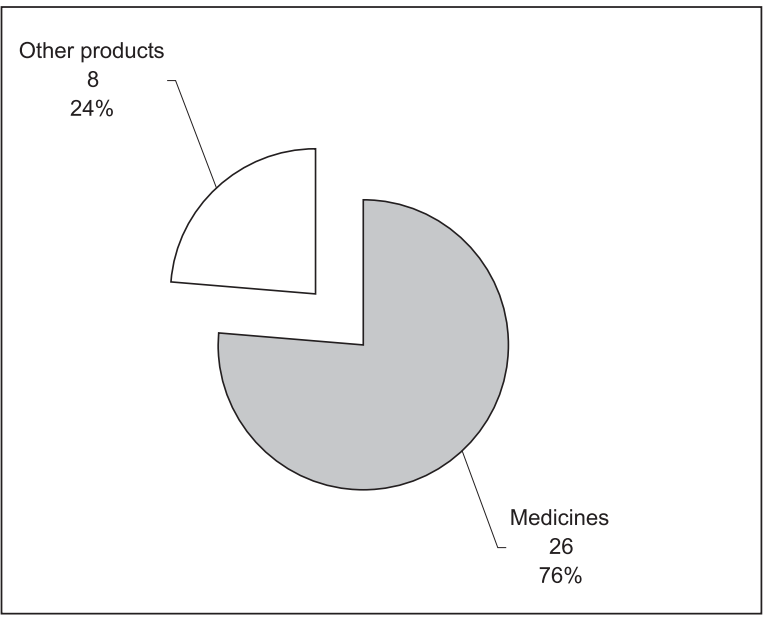

Figure 1 Medicines and other products from illegal supply

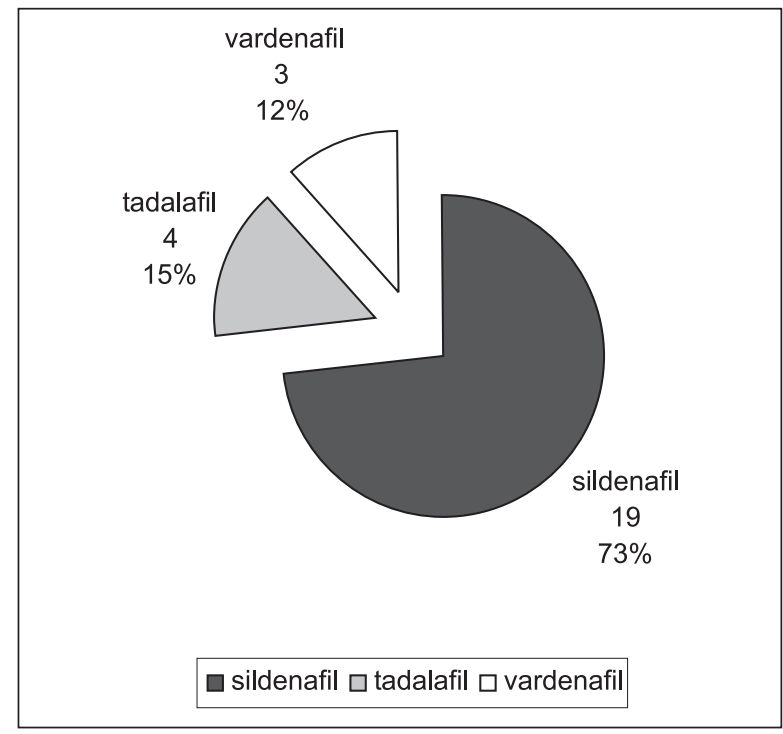

Figure 2 Medicinal products from illegal supply according to their active pharmaceutical ingredients
The purpose of this study was to determine the safety of samples identified as sildenafil and taldalafil in view of possible counterfeiting.

\section{MATERIALS AND METHODS}

The samples were submitted to the Agency as the regulatory authority by Zagreb Police Department. High performance liquid chromatography (HPLC) was used to separate sildenafil and tadalafil from their potential degradation products, process-related impurities, and formulation constituents according to manufacturer instructions. Sildenafil and tadalafil were quantified by comparing chromatographic peak areas of sample solutions with reference standard solutions. The following reference substances were used to prepare reference solutions: sildenafil citrate; Pfizer (USA), lot 0015-QSC-23 and tadalafil; Lilly (USA); lot 991085.

To determine active substances in tablets of the illegal products, we used a liquid chromatograph equipped with a column oven, a variable wavelength ultraviolet absorption detector, and a sample injection system. Sildenafil and tadalafil content in test samples was calculated as percentage of declared content of sildenafil and tadalafil per tablet.

Chromatographic conditions for sildenafil and tadalafil identification are given in Table 1.

\section{RESULTS AND DISCUSSION}

If a finished medicinal product is licensed for marketing in Croatia and the manufacturer is based outside Croatia, the distributor or the importer shall apply to the Agency for quality verification for every imported batch or for Agency's consent if its quality has been tested in the European Union. A comparison with samples licensed for marketing in Croatia (sildenafil $50 \mathrm{mg}$ tablets, sildenafil $100 \mathrm{mg}$ tablets, tadalafil $20 \mathrm{mg}$ tablets, vardenafil $10 \mathrm{mg}$ tablets, vardenafil $20 \mathrm{mg}$ tablets) showed that samples submitted by the police were not authorised for marketing in Croatia, and were most likely intended for distribution in countries other than Croatia or for an illegal drug market.

Medicinal products lacking marketing authorisation for Croatia may be imported only with a special import license issued by the Agency. Since its foundation, the 
Table 1 Chromatographic conditions for sildenafil and tadalafil identification

\begin{tabular}{|c|c|c|}
\hline & Sildenafil & Tadalafil \\
\hline Column & $\begin{array}{l}\text { Symmetry Shield RP } 18, \\
150 \mathrm{~mm} \times 3.9 \mathrm{~mm} ; 5 \mu \mathrm{m}\end{array}$ & $\begin{array}{l}\text { Symmetry Shield RP } 18, \\
150 \mathrm{~mm} \times 3.9 \mathrm{~mm} ; 5 \mu \mathrm{m}\end{array}$ \\
\hline Detector & UV $290 \mathrm{~nm}$ & UV $285 \mathrm{~nm}$ \\
\hline Column temperature & $30^{\circ} \mathrm{C}$ & $30{ }^{\circ} \mathrm{C}$ \\
\hline Injection volume & $20 \mu \mathrm{L}$ & $10 \mu \mathrm{L}$ \\
\hline Buffer & $\begin{array}{l}0.05 \mathrm{~mol} \mathrm{~L}^{-1} \text { triethylamine solution modified } \\
\text { to } \mathrm{pH} 3.0 \pm 0.05 \text { with } 1: 10 \text { diluted phosphate } \\
\text { acid or } 0.01 \mathrm{~mol} \mathrm{~L}^{-1} \mathrm{NaOH}\end{array}$ & \\
\hline Mobile phase & buffer : acetonitrile : methanol (58:17:25); & $\begin{array}{l}\text { acetonitrile : water with } 0.1 \%(\mathrm{v} / \mathrm{v}) \\
\quad \text { trifluoroacetic acid }(35: 65)\end{array}$ \\
\hline Flow rate & $1.0 \mathrm{~mL} \mathrm{~min}^{-1}$ & $1.0 \mathrm{~mL} \mathrm{~min}^{-1}$ \\
\hline
\end{tabular}

Agency has never issued a special import licence for any of the reference medicinal products $(\mathrm{C} /$ Finegra $100 \mathrm{mg}$ tablets ${ }^{1}$, Sildofil $100 \mathrm{mg}$ tablets ${ }^{2}$, Cobra 100 mg tablets ${ }^{3}$, Kamagra $^{\mathrm{TM}} 100 \mathrm{mg}$ tablets $^{4}$, Novagra forte $100 \mathrm{mg}$ tablets $^{5}$, Vega $100 \mathrm{mg}$ tablets $^{6}$, Venegra 100 $\mathrm{mg}$ tablets ${ }^{7}$, Virecta $100 \mathrm{mg}$ tablets $^{8}$, Apcalis plus 20 mg tablets $)$.

Other submitted samples not identified as medicines or medical devices are not within Agency's scope of responsibility (Figure 1). One can not discard the possibility that some of these products are classified as medicines in other countries. This group of products includes Femi- $\mathrm{X}^{10}$ tablets, manufactured by Danish Pharmaceutical Industries Ltd., that have been authorised for marketing in Croatia as a dietary supplement intended to increase libido in women. Other products, Wollust Tropfen, Erotic Fluid, and Original Inverma Yohimbinum ${ }^{11}$ were manufactured by Inverma Chemie, Germany. According to the declaration, these products are intended to increase potency and contain ginseng extract and yohimbine at very low concentrations otherwise typical of homeopathic products.

During authorisation of authentic medicines, the Agency for Medicinal Products and Medical Devices approved the manufacturer's specifications which were used in the Agency for testing the first group of drugs. The tests included verification and quantification of

\footnotetext{
${ }^{1}$ Manufacturer is not specified

${ }^{2}$ Future Pharmaceuticals

${ }^{3} \mathrm{MB} \mathrm{C}$

${ }^{4}$ Aajanta Pharma Limited

${ }^{5}$ Brown \& Burk (UK) Ltd

${ }^{6}$ Manufacturer is not specified

Anvaxx Laboratory, USA

EVA Pharma

9 MS Pharma

${ }^{10}$ Femi-X A/S

${ }^{11}$ Inverma Arzneimittel
}

the active substance against reference substance using high-performance liquid chromatography (HPLC).

The Agency does not possess any manufacturers' documentation or analytical instructions for medicines lacking marketing authorisation in Croatia. As, according to the declaration, the medicines from the second group contained the same active substance as the authorised medicinal products, we used the same methods as for licensed finished medicinal products to verify and quantify them. As not all submitted batches had enough samples to meet manufacturer's instructions for analysis, we tested only two samples per batch, and the results were not statistically analysed.

Sildenafil samples were prepared in accordance with the procedure for Viagra ${ }^{\circledR}$ tablets manufactured by Pfizer. Two samples containing one tablet each were tested from each batch (Table 2, Figure 3).

Tadalafil samples, again two per batch, were prepared by combining three individual tablets in a $250 \mathrm{~mL}$ volumetric flask and processing them in accordance with the procedure for Cialis tablets manufactured by Lilly (Table 2, Figure 4).

The presence of the active substance has been confirmed in all tested samples, so in qualitative terms all samples conformed to declarations. Table 2 and Figure 5 show the quantitative composition in the tested samples of the first and second group of medicines. Samples labelled 1 (batch T/33), 3 (batchT/33), 5 (batch 4), 6 (batch M0016J), 10 (batch T-070235), 12 (batch T-070544), 15 (batch 314833201), 16 (batch 832718474), 17 (batch 504830028) 20 (batch 070356), 21 (batch 05668), and 22 (batch T 378 5), contained the active substance outside the acceptable $95 \%$ to $105 \%$ margin of deviation from the declared value. These deviations confirmed drug defects and the suspicion of counterfeit (Figure 6). 


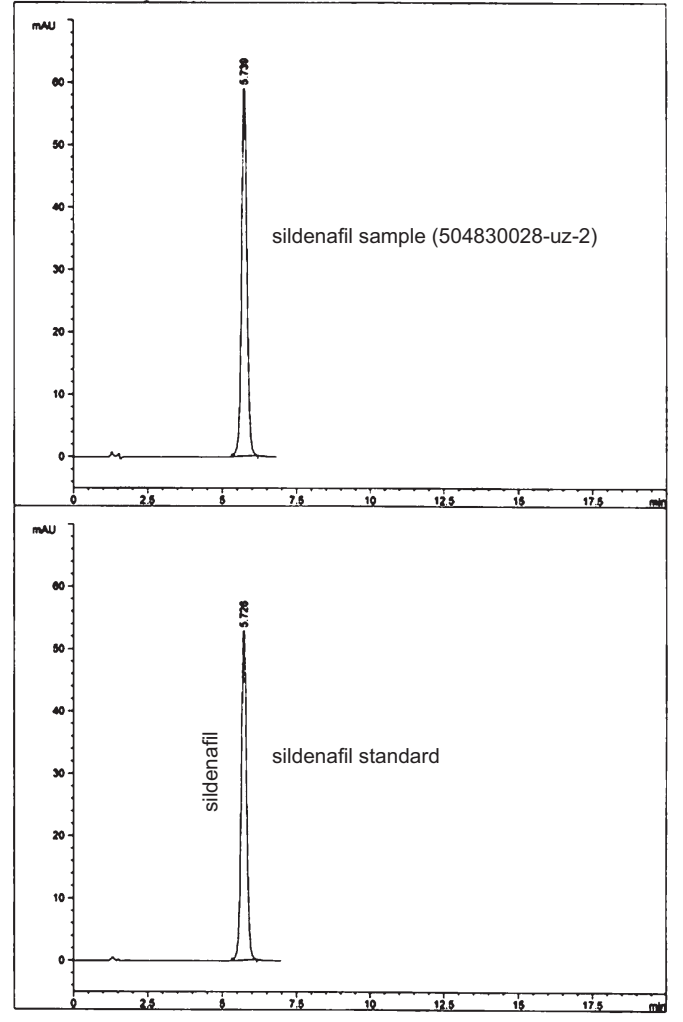

Figure 3 A typical chromatogram of sildenafil sample and reference standard

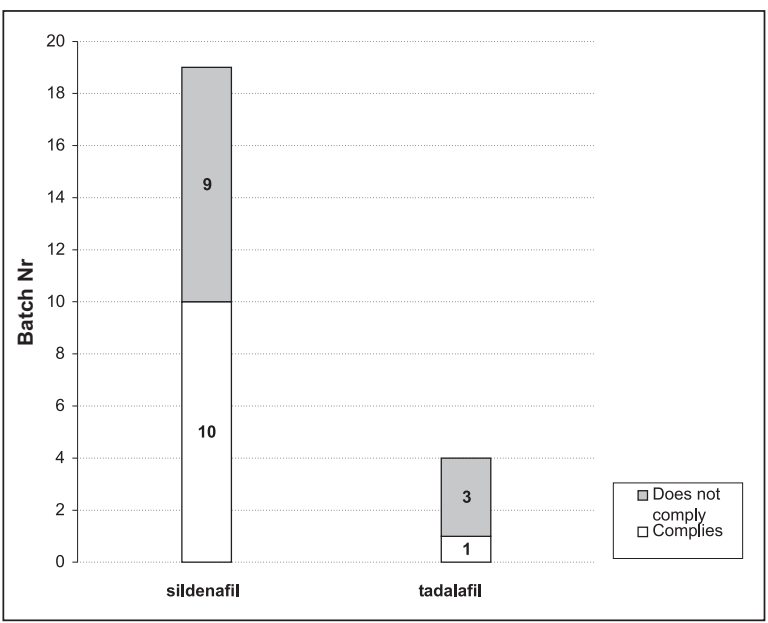

Figure 5 Test results of sildenafil and tadalafil tablets

\section{CONCLUSION}

Even if test results fall within acceptable margins, one can safely verify the authenticity of a medicine only after receiving manufacturer's opinion based on complete batch data. Manufacturers alone possess complete data and in case of suspicion can run a

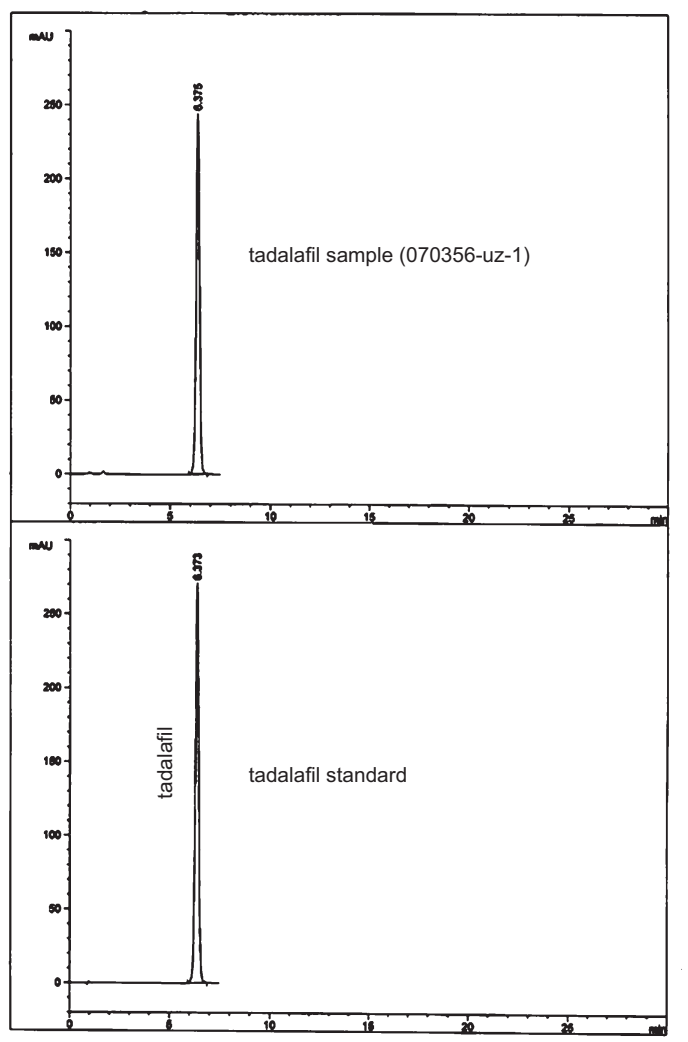

Figure 4 A typical chromatogram of tadalafil sample and reference standard

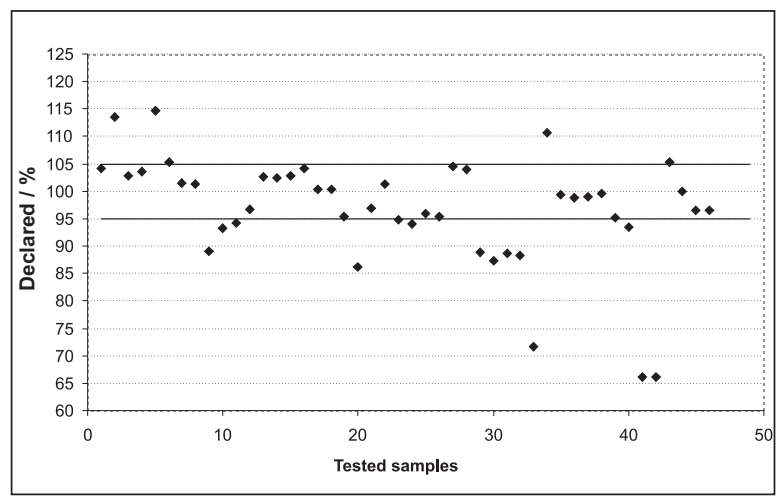

Figure 6 Deviation from active substance content in tablet samples

series of additional target tests in their laboratories, including additional tests for purity profiles of active substances, excipients used in the finished drug product, type and material of primary and secondary packaging, or the quality and credibility of printing. These additional tests can make it easier and quicker to see whether a drug is authentic or fake; indeed, they are sometimes the only means to do this. Sufficiently discriminating analytical techniques are also needed, 
Table 2 Test results of sildenafil and tadalafil containing tablets

\begin{tabular}{|c|c|c|c|c|c|c|c|}
\hline \multirow[b]{2}{*}{$\begin{array}{l}\text { Sample } \\
\text { no. }\end{array}$} & \multirow[b]{2}{*}{$\begin{array}{c}\text { Proprietary } \\
\text { name }\end{array}$} & \multirow[b]{2}{*}{ Manufacturer } & \multirow{2}{*}{$\begin{array}{c}\text { Active } \\
\text { substance / } \\
\text { dose }\end{array}$} & \multirow[b]{2}{*}{ Package } & \multirow[b]{2}{*}{ Batch } & \multicolumn{2}{|c|}{ Content } \\
\hline & & & & & & $\begin{array}{l}\text { mg per } \\
\text { tablet }\end{array}$ & $\begin{array}{c}\text { Deviation } \\
/ \%\end{array}$ \\
\hline 1 & Sildofil 100 & $\begin{array}{c}\text { Future } \\
\text { Pharmaceuticals }\end{array}$ & $\begin{array}{l}\text { sildenafil / } \\
100 \mathrm{mg}\end{array}$ & $\begin{array}{l}4 \text { tablets in a blister } \\
\text { packaged in a carton }\end{array}$ & $\mathrm{T} / 33$ & $\begin{array}{l}104.1 \\
113.6\end{array}$ & $\begin{array}{l}104.1 \\
113.6\end{array}$ \\
\hline 2 & Sildofil 100 & $\begin{array}{c}\text { Future } \\
\text { Pharmaceuticals }\end{array}$ & $\begin{array}{l}\text { sildenafil / } \\
100 \mathrm{mg}\end{array}$ & $\begin{array}{l}4 \text { tablets in a blister } \\
\text { packaged in a carton }\end{array}$ & $\mathrm{T} / 20$ & $\begin{array}{l}102.9 \\
103.6\end{array}$ & $\begin{array}{l}102.9 \\
103.6\end{array}$ \\
\hline 3 & Sildofil 100 & $\begin{array}{c}\text { Future } \\
\text { Pharmaceuticals }\end{array}$ & $\begin{array}{l}\text { sildenafil / } \\
100 \mathrm{mg}\end{array}$ & 4 tablets in a blister & $\mathrm{T} / 33$ & $\begin{array}{l}114.7 \\
105.3\end{array}$ & $\begin{array}{l}114.7 \\
105.3\end{array}$ \\
\hline 4 & $\mathrm{C} /$ Finegra & not specified & $\begin{array}{l}\text { sildenafil / } \\
100 \mathrm{mg}\end{array}$ & 4 tablets in a blister & 2036 & $\begin{array}{l}101.4 \\
101.2\end{array}$ & $\begin{array}{l}101.4 \\
101.2\end{array}$ \\
\hline 5 & Cobra $100 \mathrm{mg}$ & MB C & $\begin{array}{l}\text { sildenafil / } \\
100 \mathrm{mg}\end{array}$ & 4 tablets in a blister & 4 & $\begin{array}{l}89.1 \\
93.3\end{array}$ & $\begin{array}{l}89.1 \\
93.3\end{array}$ \\
\hline 6 & $\begin{array}{l}\text { Kamagra*Trade } \\
\text { Mark } 100 \text { mg }\end{array}$ & $\begin{array}{c}\text { Aajanta Pharma } \\
\text { Limited }\end{array}$ & $\begin{array}{l}\text { sildenafil / } \\
100 \mathrm{mg}\end{array}$ & $\begin{array}{l}4 \text { tablets in a blister } \\
\text { packaged in a carton }\end{array}$ & M0016J & $\begin{array}{l}94.2 \\
96.7\end{array}$ & $\begin{array}{l}94.2 \\
96.7\end{array}$ \\
\hline 7 & Novagra-Forte & $\begin{array}{c}\text { Brown \& Burk (UK) } \\
\text { Ltd. }\end{array}$ & $\begin{array}{l}\text { sildenafil / } \\
100 \mathrm{mg}\end{array}$ & $\begin{array}{l}4 \text { tablets in a blister } \\
\text { packaged in a carton }\end{array}$ & $\begin{array}{c}\text { NVFH } \\
0028\end{array}$ & $\begin{array}{l}102.6 \\
102.4\end{array}$ & $\begin{array}{l}102.6 \\
102.4\end{array}$ \\
\hline 8 & Novagra-Forte & $\begin{array}{c}\text { Brown \& Burk (UK) } \\
\text { Ltd. }\end{array}$ & $\begin{array}{l}\text { sildenafil / } \\
100 \mathrm{mg}\end{array}$ & $\begin{array}{l}4 \text { tablets in a blister } \\
\text { packaged in a carton }\end{array}$ & $\begin{array}{c}\text { NVFH } \\
0030\end{array}$ & $\begin{array}{l}102.9 \\
104.2\end{array}$ & $\begin{array}{l}102.9 \\
104.2\end{array}$ \\
\hline 9 & Novagra-Forte & $\begin{array}{l}\text { Brown \& Burk (UK) } \\
\text { Ltd. }\end{array}$ & $\begin{array}{l}\text { sildenafil / } \\
100 \mathrm{mg}\end{array}$ & $\begin{array}{l}4 \text { tablets in a blister } \\
\text { packaged in a carton }\end{array}$ & $\begin{array}{c}\text { NVFH } \\
0031\end{array}$ & $\begin{array}{l}100.3 \\
100.4\end{array}$ & $\begin{array}{l}100.3 \\
100.4\end{array}$ \\
\hline 10 & Vega 100 & not specified & $\begin{array}{l}\text { sildenafil / } \\
100 \mathrm{mg}\end{array}$ & 4 tablets in a blister & $\mathrm{T}-070235$ & $\begin{array}{l}95.3 \\
86.1\end{array}$ & $\begin{array}{l}95.3 \\
86.1\end{array}$ \\
\hline 11 & Vega 100 & not specified & $\begin{array}{l}\text { sildenafil / } \\
100 \mathrm{mg}\end{array}$ & 4 tablets in a blister & $\mathrm{T}-070237$ & $\begin{array}{c}96.9 \\
101.2\end{array}$ & $\begin{array}{c}96.9 \\
101.2\end{array}$ \\
\hline 12 & Vega 100 & not specified & $\begin{array}{l}\text { sildenafil / } \\
100 \mathrm{mg}\end{array}$ & 4 tablets in a blister & T-070544 & $\begin{array}{l}94.8 \\
94.1\end{array}$ & $\begin{array}{l}94.8 \\
94.1\end{array}$ \\
\hline 13 & Vega 100 Asia & not specified & $\begin{array}{l}\text { sildenafil / } \\
100 \mathrm{mg}\end{array}$ & 4 tablets in a blister & T-2106 & $\begin{array}{l}96.0 \\
95.4\end{array}$ & $\begin{array}{l}96.0 \\
95.4\end{array}$ \\
\hline 14 & Venegra & $\begin{array}{c}\text { Anvaxx Laboratory, } \\
\text { USA }\end{array}$ & $\begin{array}{l}\text { sildenafil / } \\
100 \mathrm{mg}\end{array}$ & $\begin{array}{l}4 \text { tablets in a blister } \\
\text { packaged in a carton }\end{array}$ & PO226C & $\begin{array}{l}104.6 \\
103.9\end{array}$ & $\begin{array}{l}104.6 \\
103.9\end{array}$ \\
\hline 15 & $\begin{array}{l}\text { VIAGRA } 100 \\
\text { mg }\end{array}$ & Pfizer Inc. & $\begin{array}{l}\text { sildenafil / } \\
100 \mathrm{mg}\end{array}$ & $\begin{array}{l}4 \text { tablets in a blister } \\
\text { packaged in a carton }\end{array}$ & 314833201 & $\begin{array}{l}88.8 \\
87.3\end{array}$ & $\begin{array}{l}88.8 \\
87.3\end{array}$ \\
\hline 16 & $\begin{array}{l}\text { VIAGRA }^{\circledR} 100 \\
\text { mg }\end{array}$ & Pfizer USA & $\begin{array}{l}\text { sildenafil / } \\
100 \mathrm{mg}\end{array}$ & $\begin{array}{l}4 \text { tablets in a blister } \\
\text { packaged in a carton }\end{array}$ & 832718474 & $\begin{array}{l}88.6 \\
88.3\end{array}$ & $\begin{array}{l}88.6 \\
88.3\end{array}$ \\
\hline 17 & VIAGRA $50 \mathrm{mg}$ & Pfizer Inc. & $\begin{array}{l}\text { sildenafil / } \\
50 \mathrm{mg}\end{array}$ & $\begin{array}{l}4 \text { tablets in a blister } \\
\text { packaged in a carton }\end{array}$ & 504830028 & $\begin{array}{l}35.8 \\
55.4\end{array}$ & $\begin{array}{c}71.7 \\
110.7\end{array}$ \\
\hline 18 & Virecta & EVA Pharma & $\begin{array}{l}\text { sildenafil / } \\
100 \mathrm{mg}\end{array}$ & $\begin{array}{l}3 \text { tablets in a blister } \\
\text { packaged in a carton }\end{array}$ & 703291 & $\begin{array}{l}99.4 \\
98.9\end{array}$ & $\begin{array}{l}99.4 \\
98.9\end{array}$ \\
\hline 19 & Virecta & EVA Pharma & $\begin{array}{l}\text { sildenafil / } \\
100 \mathrm{mg}\end{array}$ & $\begin{array}{l}3 \text { tablets in a blister } \\
\text { packaged in a carton }\end{array}$ & 704391 & $\begin{array}{l}99.0 \\
99.5\end{array}$ & $\begin{array}{l}99.0 \\
99.5\end{array}$ \\
\hline 20 & Apcalis Plus 20 & MS Pharma & $\begin{array}{l}\text { tadalafil / } \\
20 \mathrm{mg}\end{array}$ & $\begin{array}{l}2 \times 2 \text { tablets in a blister } \\
\text { packaged in a carton }\end{array}$ & 070356 & $\begin{array}{l}19.0 \\
18.7\end{array}$ & $\begin{array}{l}95.2 \\
93.5\end{array}$ \\
\hline 21 & Cialis $^{\circledR} 20 \mathrm{mg}$ & Lilly ICOS & $\begin{array}{l}\text { tadalafil / } \\
20 \mathrm{mg}\end{array}$ & $\begin{array}{l}2 \times 2 \text { tablets in a blister } \\
\text { packaged in a carton }\end{array}$ & 05668 & $\begin{array}{l}13.2 \\
13.2\end{array}$ & $\begin{array}{l}66.1 \\
66.1\end{array}$ \\
\hline 22 & Cyvel 20 mg & $\begin{array}{c}\text { Barakat } \\
\text { Pharmaceutical } \\
\text { Industries }\end{array}$ & $\begin{array}{l}\text { tadalafil / } \\
20 \mathrm{mg}\end{array}$ & 2 tablets in a blister & Т 3785 & $\begin{array}{l}21.1 \\
20.0\end{array}$ & $\begin{array}{l}105.4 \\
100.0\end{array}$ \\
\hline 23 & Tadlis 20 & Orient Pharma & $\begin{array}{l}\text { tadalafil / } \\
20 \mathrm{mg}\end{array}$ & $\begin{array}{l}2 \times 2 \text { tablets in a blister } \\
\text { packaged in a carton }\end{array}$ & 702099 & $\begin{array}{l}19.3 \\
19.3 \\
\end{array}$ & $\begin{array}{l}96.6 \\
96.6 \\
\end{array}$ \\
\hline
\end{tabular}


such as the near infrared spectroscopy (NIR), which is a mandatory method for fingerprint identification of medicinal products by manufacturers (19-21). Such tests are designed to identify the manufacturing site and deviations from manufacturer's standards for each manufactured batch.

\section{REFERENCES}

1. Zarocostas J. WHO to set up international task force on counterfeit drugs. BMJ 2006;332:444.

2. Thompson CA. Counterfeit drugs arise from various sources. Am J Health Syst Pharm 2003;60:1506-11.

3. Newton PN, White NJ, Rozendaal JA, Green MD. Murder by fake drugs: Time for international action. BMJ 2002;324:8001 .

4. Korcok M. Counterfeit drugs latest public health hazard in US. CMAJ 2003;169:223.

5. Aldhous P. Counterfeit pharmaceuticals: Murder by medicine. Nature 2005;434:132-6.

6. Pincock S. WHO tries to tackle problem of counterfeit medicines in Asia. BMJ 2003;327:1126.

7. Chauvé M. La lutte contre la contrefaçon de médicaments en Afrique: expériences et rôle des pharmaciens [The fight against counterfeit medicines in Africa: experience and role of pharmacists, in French]. C R Biol 2008;331:982-5.

8. Caudron JM, Ford N, Henkens M, Macé C, Kiddle-Monroe R, Pinel J. Substandard medicines in resource-poor settings: a problem that can no longer be ignored. Trop Med Int Health 2008; 13:1062-72.
9. Schofield J. Counterfeit pharmaceuticals flood Russian market. BMJ 2001;322:156.

10. Tomlinson R. China cracks down on counterfeit medicines. BMJ 1999;318:624.

11. Gibson L. Counterfeits of impotence drug appear in the United Kingdom. BMJ 2004;329:532.

12. Roger B, Boateng K. Bad medicine in the market. World Hosp Health Serv 2007;43:17-21.

13. Amon JJ. Dangerous medicines: Unproven AIDS cures and counterfeit antiretroviral drugs. Global Health 2008;4:5.

14. Newton PN, Green MD, Fernández FM, Day NP, White NJ. Counterfeit anti-infective drugs. Lancet Infect Dis 2006;6:602-13.

15. Mayor S. EU should strengthen protection against fake drugs, UK report says. BMJ 2006;333:1090.

16. Deisingh AK. Pharmaceutical counterfeiting. Analyst 2005;130:271-9.

17. Jack A. Counterfeit medicines: Bitter pills. BMJ 2007;335:1120-1

18. Gibson L. Drug regulators study global treaty to tackle counterfeit drugs. BMJ 2004;328:486.

19. Maurin JK, Pluciński F, Mazurek AP, Fijałek Z. The usefulness of simple X-ray powder diffraction analysis for counterfeit control - the Viagra example. J Pharm Biomed Anal 2007;43:1514-8.

20. de Veij M, Deneckere A, Vandenabeele P, de Kaste D, Moens 1. Detection of counterfeit Viagra with Raman spectroscopy. J Pharm Biomed Anal 2008;46:303-9.

21. Vredenbregt MJ, Blok-Tip L, Hoogerbrugge R, Barends DM, de Kaste D. Screening suspected counterfeit Viagra and imitations of Viagra with near-infrared spectroscopy. J Pharm Biomed Anal 2006;40:840-9. 


\section{Sažetak \\ IDENTIFIKACIJA KRIVOTVORENIH LIJEKOVA ZA EREKTILNU DISFUNKCIJU IZ ILEGALNOG LANCA OPSKRBE}

Pojava krivotvorenih lijekova u lancima opskrbe globalni je javnozdravstveni problem koji može imati ozbiljnih posljedica za pacijenta. Krivotvoreni lijekovi ne zadovoljavaju propisane standarde kakvoće te su u svrhu prijevare drugačije deklarirani u odnosu na njihov stvarni sastav i/ili podrijetlo. Mogu biti generički, ili inovativni, mogu sadržavati ispravne sastojke, ali pogrešno pakiranje, pogrešne sastojke, ne sadržavati djelatnu tvar ili ne sadržavati dovoljnu količinu djelatne tvari. U Hrvatskoj do sada nisu zabilježeni slučajevi krivotvorenih lijekova, ali je Agencija za lijekove i medicinske proizvode od policijske uprave Zagrebačke zaprimila 34 uzorka lijeka i ostalih proizvoda u svrhu ispitivanja. Od lijekova, radilo se o lijekovima za erektilnu disfunkciju sildenafilu, tadalafilu i vardenafilu. 23 uzorka tableta koji nemaju odobrenje za stavljanje lijeka u promet u Hrvatskoj ispitana su metodom tekućinske kromatografije visokog učinka (HPLC) na deklarirani sadržaj sildenafila i tadalafila. Ustanovljeno je da uzorci pod oznakama 1 (serija T/33), 3 (serijaT/33), 5 (serija 4), 6 (serija M0016J), 10 (serija T-070235), 12 (serija T-070544), 15 (serija 314833201), 16 (serija 832718474), 17 (serija 504830028) koji su sadržavali sildenafil te uzorci 20 (serija 070356), 21 (serija 05668), 22 (serija T 378 5) i 23 (702099) koji su sadržavali tadalafil, ne sadržavaju djelatnu tvar unutar prihvatljivih granica odstupanja od $95 \%$ do $105 \%$ od deklarirane vrijednosti. Iako se ne može sa sigurnosti za većinu uzoraka reći da se radi o krivotvorini, ipak se može govoriti o sumnji na krivotvorinu, a cjeloviti zaključak može se izvesti jedino uz suradnju i mišljenje proizvođača te provedbom dodatnih laboratorijskih ispitivanja.

KLJUČNE RIJEČI: farmaceutski kriminal, HPLC, provjera kakvoće, sildenafil, tadalafil, vardenafil

\section{CORRESPONDING AUTHOR:}

Siniša Tomić, $\mathrm{PhD}$

Agency for Medicinal Products and Medical Devices

Ksaverska cesta 4, HR-10000 Zagreb, Croatia

E-mail:sinisa.tomic@almp.hr 